\title{
Effect of Temperature in the Evolution of Ni/Al Nanolayers
}

\author{
A. S. Ramos, ${ }^{*}$ S. Simões, ${ }^{* *}$ Rosa Calinas, ${ }^{*}$ and F. Viana ${ }^{* *}$
}

* ICEMS, Departamento de Engenharia Mecânica, Faculdade de Ciências e Tecnologia, Universidade de Coimbra, R. Luís Reis Santos, 3030-788 Coimbra, Portugal

** Departamento de Engenharia Metalúrgica e Materiais, GMM/IMAT, Faculdade de Engenharia, Universidade do Porto, Rua Dr. Roberto Frias, 4200-465 Porto, Portugal

Nickel aluminides can be formed by thermal annealing of alternate layers of $\mathrm{Ni}$ and $\mathrm{Al}$ that react exothermically. These metals have medium/high energy of mixing and when in adiabatic conditions the reaction could become self-propagating [1]. The first phase to form in $\mathrm{Ni} / \mathrm{Al}$ reactions has been widely investigated, but there is no consensus regarding this subject. Depending on the overall chemical composition and modulation period of the multilayer, and on the processing history, the first phase to form could be $\mathrm{NiAl}_{3}, \mathrm{NiAl}$ and $\mathrm{Ni}_{2} \mathrm{Al}_{9}[2,3]$.

$\mathrm{Ni} / \mathrm{Al}$ multilayer thin films with nanometric period were deposited onto stainless steel substrates by d.c. magnetron sputtering using nickel and aluminium pure targets. The morphology of the films was evaluated by scanning electron microscopy (SEM). The structural evolution with temperature of the Ni/Al multilayer thin films was studied by in situ hot X-ray diffraction (XRD) and differential scanning calorimetry (DSC).

The deposition procedure gives rise to a nanometric multilayer structure (figure 1), where $\mathrm{Ni}$ (bright) and $\mathrm{Al}$ (dark) layers can be identified by scanning electron microscopy. The $\mathrm{Ni}$ and $\mathrm{Al}$ single layer thickness is a function of the time that the substrates are in front of each target during one rotation; but also depends on the diffusion that might occur during the deposition process. The deposition conditions selected resulted in a multilayer thin film with a bilayer thickness close to $30 \mathrm{~nm}$ that consists of two solid solutions, one Ni-rich and the other Al-rich. During heat treatment, the $\mathrm{Ni}$ and $\mathrm{Al}$ of the nanometric multilayer thin films undergo interdiffusion and reaction with the formation of intermetallic phases. After annealing at increasing temperatures in a furnace, in a XRD hot chamber and in a DSC equipment, the ultimate phase is always B2-NiAl. However, according to the XRD and DSC results an intermediate phase indexed as $\mathrm{NiAl}_{3}$ is formed. The structural evolution was also followed by in situ SEM in order to observe the morphological changes occurring during reaction at increasing temperatures. As the temperature increases the layered structure tends to vanish, although after heat treatment up to $700^{\circ} \mathrm{C}$ it does not completely disappear as shown in figure 2. On the other hand, the effect of time also plays an important role on the reaction between $\mathrm{Ni}$ and $\mathrm{Al}$. In fact, after annealing at $325^{\circ} \mathrm{C}$ during $1 \mathrm{~h} 30 \mathrm{~min}$ a layered structure is still visible but after $3 \mathrm{~h}$ at $425^{\circ} \mathrm{C}$ the films have completely reacted and consist of a NiAl single phase. Summing up, Ni/Al multilayers with a $30 \mathrm{~nm}$ period react at rather low temperatures forming $\mathrm{NiAl}_{3}$ and $\mathrm{NiAl}$ intermetallic compounds depending on the heat treatment temperature and time.

Acknowledgements

This work was supported by Fundação para a Ciência e Tecnologia through the project PTDC/CTM/69645/2006 and under contracts BPD/6771/2001 and BD/30371/2006, co-financed by European Union fund FEDER. 


\section{References}

[1] T. P. Weihs, "Self-propagating reactions in multilayer materials" in Handbook of Thin Films Process Technology, Institute of Physics Publishing, Bristol, 1998, Part F.

[2] K. Barmak, C. Michaelsen and G. Lucadamo, J. Mater. Res. 12 (1997) 133.

[3] K. J. Blobaum, D. Van Heerden, A. J. Gavens and T. P. Weihs, Acta Mater. 51 (2003) 3884.

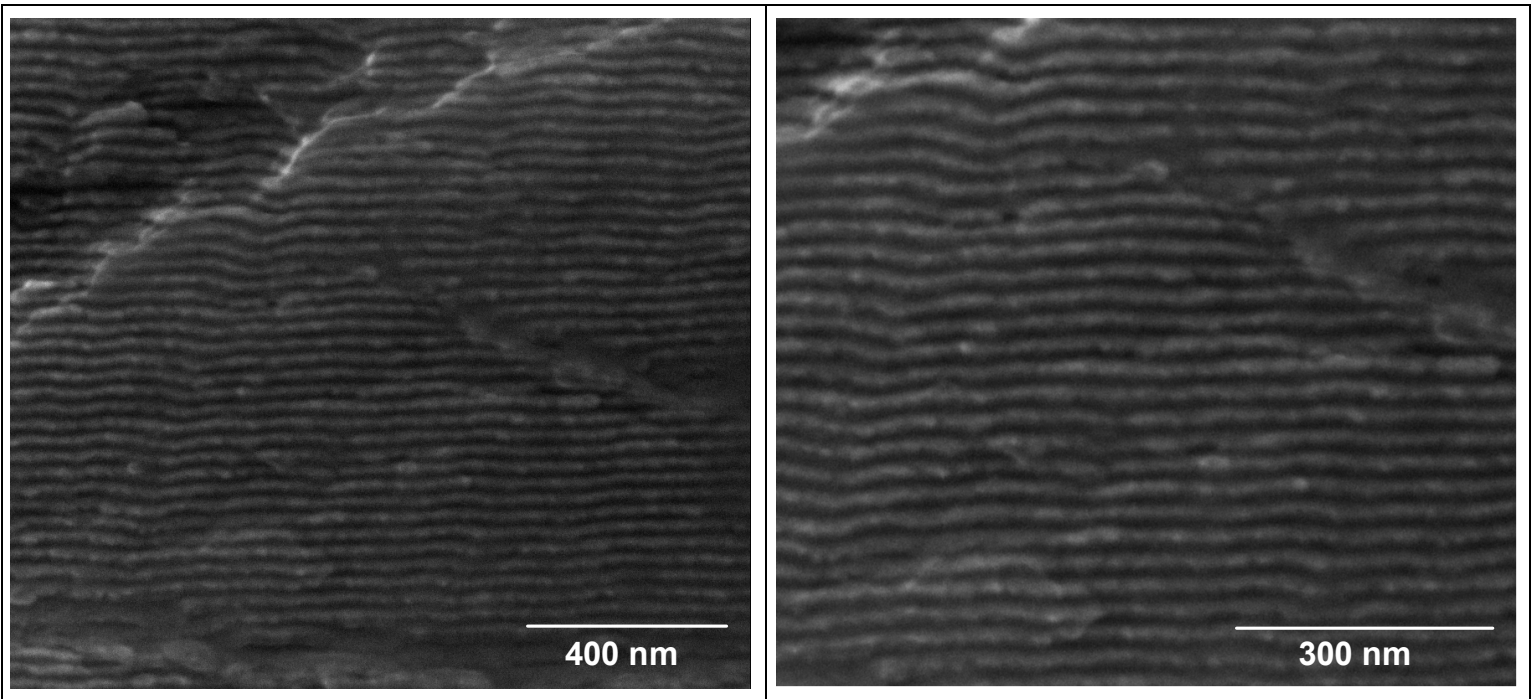

Figure 1-SEM cross-section images of an as-deposited Ni/Al multilayer thin film.
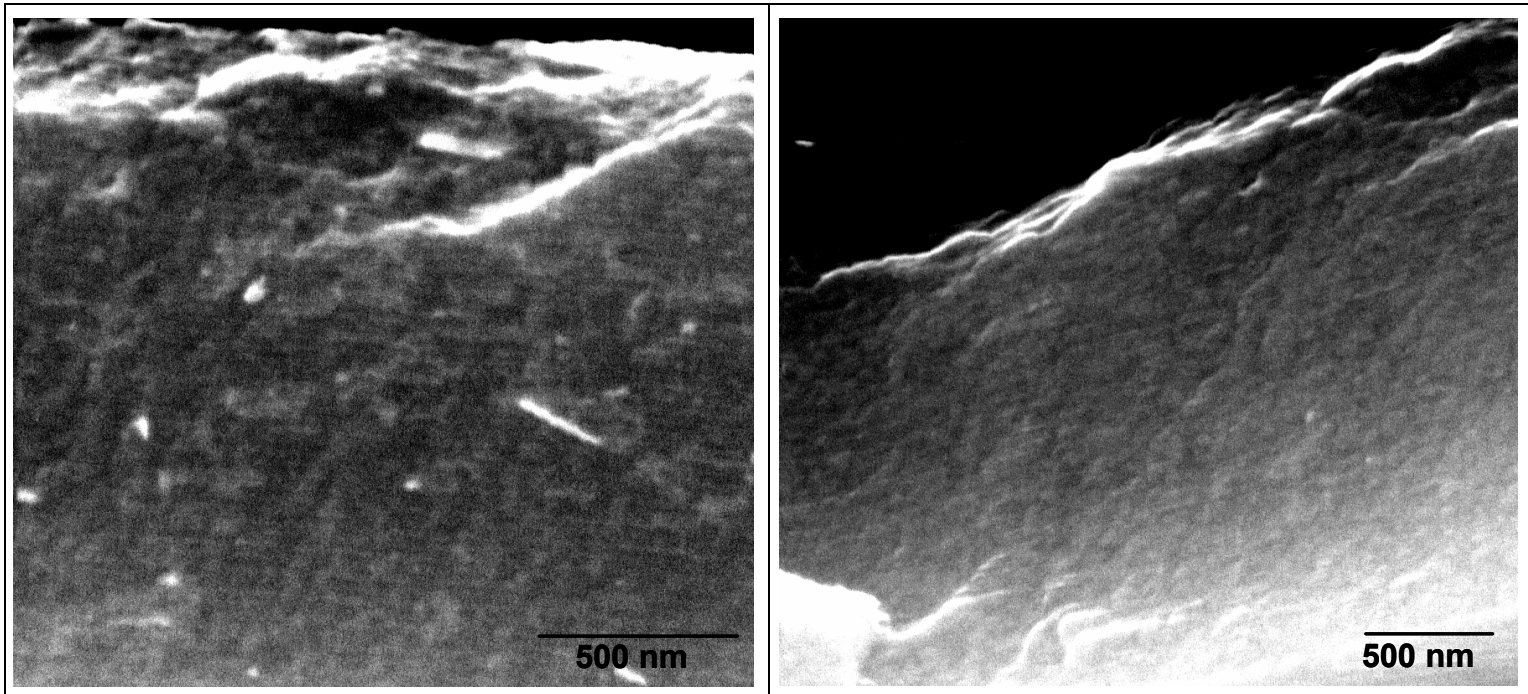

Figure 2 - SEM cross-section images of Ni/Al thin films in situ heat treated at a) $600^{\circ} \mathrm{C}$ and b) $700^{\circ} \mathrm{C}$. 